\title{
Development of a radiation based heat model for satellite attitude determination
}

\author{
A. Labibian ${ }^{\mathrm{a}}$, S.H. Pourtakdoust ${ }^{\mathrm{b}, *}$, A. Alikhani $^{\mathrm{c}}$, H. Fourati ${ }^{\mathrm{d}}$ \\ a Aerospace Research Institute and Sharif University of Technology, Tehran, 14665 834, Iran \\ b Center for Research and Development in Space Science and Technology, Sharif University of Technology, Tehran, 145888 9694, Iran \\ c Aerospace Research Institute, Ministry of Science, Research and Technology, Tehran, 14665 834, Iran \\ ${ }^{\mathrm{d}}$ University Grenoble Alpes, CNRS, GIPSA-Lab, Grenoble 38400, France
}

\section{Keywords:}

Attitude determination

Heat attitude model

Net heat flux

Temperature data

\begin{abstract}
A B S T R A C T
This paper is focused on the development and verification of a heat attitude model (HAM) for satellite attitude determination. Within this context, the Sun and the Earth are considered as the main external sources of radiation that could effect the satellite surface temperature changes. Assuming that the satellite orbital position (navigational data) is known, the proposed HAM provides the satellite surface temperature with acceptable accuracy and also relates the net heat flux (NHF) of three orthogonal satellite surfaces to its attitude via the inertial to satellite transformation matrix. The proposed HAM simulation results are verified through comparison with commercial thermal analysis tools. The proposed HAM has been successfully utilized in some researches for attitude estimation, and further studies for practical implementations are still ongoing.
\end{abstract}

\section{Introduction}

Attitude determination (AD) of satellites has been an important issue since early developments of the first space mission, and it is still the subject of many scientific and applied researches. Proper knowledge of attitude is required for pointing capabilities via the satellite Attitude Control System (ACS). The history of research in AD goes back to 1965, when G. Wahba initially proposed her method of attitude estimation [1]. In general, when the AD of satellites is studied, the following three main parts need to be addressed: attitude representation, attitude sensors and estimation algorithms. There exists many researches on each of the above mentioned parts, under the so called AD problem. For the attitude representation, Euler angles, quaternion and Gibbs Vector are widely used. Conventional attitude sensors include magnetometer, gyroscope, Sun sensor, Earth sensor, as well as star tracker that can either be used separately [2,3] or in combination [4-6]. Sensors choice is usually dictated by mission statements and/or is dependent on the desired autonomy and accuracy. Estimation algorithms are also widespread and still subject of many current researches. Extended Kalman Filter (EKF) is known as one of the most popular

\footnotetext{
* Corresponding author.

E-mail addresses: a.labibian@gmail.com (A. Labibian), pourtak@sharif.edu (S.H. Pourtakdoust), Aalikhani@Ari.ac.ir (A. Alikhani), hassen.fourati@gipsa-lab.grenoble-inp.fr (H. Fourati)
}

techniques for attitude estimation in many applications. EKF uses nonlinear attitude equations in a forward estimation process and updates the estimated states in a recursive way [7-10]. Sometimes, some constraints are a critical issue, then the structure of EKF can be modified in order to satisfy the constraints. Multiplicative EKF is developed in that case, with a fairly acceptable performance [11,12]. Unscented Kalman Filter (UKF) and particle filter (PF) are among other alternatives for EKF. Although UKF increases the computation burden, it is suitable for applications involving complex measurement equations, and its performance is better than those of EKF [13-17]. PF has emerged as a new filter structure that is based on sequential Monte Carlo Simulation (MCS) in which distributions are approximated by weighted particles [18-21].

During last years, the thermal modelling and testing tools and techniques used in satellite programs have become a major area of research. Multiple thermal nodes are considered and numerical methods are used to solve the resulting thermal coupled nonlinear partial differential equations [22,23]. Since the computational cost of these methods is high, there are analytical schemes that are less costly to use depending on the application [24-26]. Further, analytical single node thermal models can also produce acceptable results [27]. Within this framework, there are some recent studies involving utility of temperature data for flux computation as primary step in $\mathrm{AD}$ [28], where limitations, resolution and accuracies of thermal data for heat flux determination are analyzed. Heat flux for $\mathrm{AD}$ is proposed in [29], where a basic Levenberg-Marquardt 


\section{Nomenclature}

Upper case

A surface area

C transformation matrix

$F_{h} \quad$ shape factor parallel to relative position vector

$F_{v} \quad$ shape factor perpendicular to relative position vector

G Earth flux

F view factor

Q heat

Q Sun direct Sun radiation heat

$Q_{\text {Earth }}$ Earth emitted heat

$Q_{\text {albedo }}^{\prime}$ albedo emitted heat

$Q_{\text {albedo }}$ absorbed albedo emitted heat

$Q_{\text {lost }}$ lost heat to deep space

$S$ solar radiation flux

$T$ temperature

Lower case

c specific heat capacity

$f_{a} \quad$ Albedo factor

$m$ surface mass

$n \quad$ normal to surface

$r \quad$ position

Greek $\alpha \quad$ absorptivity

$\varepsilon \quad$ average emissivity

$\beta \quad$ angle between normal to the surface and relative position vectors

$\varphi, \theta, \psi \quad$ Euler angles

$\theta_{A} \quad$ angle between Sun-Earth vector and albedo reflection

$\sigma \quad$ Stefan-Boltzmann constant

$\omega \quad$ angular velocity

Overscript

$\rightarrow \quad$ vector

Subscripts

b black body

$i$ number of surface

nb nonblack surface

Superscripts

b body

e emission

I inertia

$r \quad$ net exchange

$s \quad$ Sun
(LM) deterministic least square algorithm is used. A major drawback of the LM algorithm is its inability for noise filtering that affects the attitude accuracy. In addition, the performance of the proposed HAM for attitude estimation is also demonstrated via EKF and UKF using MCS [30]. Moreover, experimental validation of the proposed HAM has been investigated [31], where a cubic laboratory satellite is used for testing within a vacuum chamber lit by a Sun simulator. There is also a commercial sensor system called Advanced Coarse Earth Sun Sensor (ACESS) [32]. It can provide an omnidirectional coarse estimation of the Earth and Sun position vector in the satellite reference frame. A combination of six ACESS packs (a total of 36 thermistors) is used to give the satellite navigation data. However, the proposed HAM requires only three non-contact thermopiles (NCT) for AD. A basic part of most estimation algorithms is the measurement model that is often a nonlinear function relating the measured data to variables whose proper estimates are needed. Therefore, the key contribution of this paper is focused on development of a novel measurement model for $\mathrm{AD}$, namely the HAM. This model is based on the net heat flux (NHF) absorbed by three orthogonal, properly insulated, surfaces of a satellite. For a typical Earth-orbiting satellite, Sun and Earth are the main heat sources to be considered. As a result, the direct Sun radiation, the Earth flux and its albedo are the major means of heat exchange with the satellite surfaces. In order to establish a suitable HAM, later for estimation process, we need to establish a relation between the NHF of each satellite surface (measurements) and the satellite attitude (states).

In this paper, we represent each surface's NHF in terms of the components of the normal to surface unit vectors using the concept of view factors. Subsequently, the knowledge of satellite relative position vectors with respect to Sun and Earth is used to relate the satellite body system to the Earth Centred Inertial (ECI) system, which leads to establish the inertial satellite attitude. The proposed HAM is verified using thermal analysis software. The satellite attitude dynamics is useful in this study to simulate the satellite attitude behaviour in orbit. Therefore, we demonstrate effectiveness of the surface temperatures resulting from the HAM for an

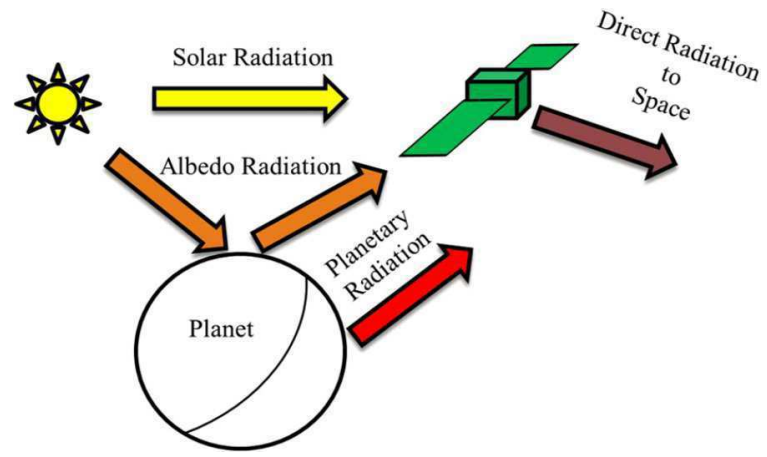

Fig. 1. Thermal environment of a typical spacecraft [33].

Earth-orbiting satellite whose attitude knowledge is simulated (or known).

This paper is organized as follows: Section 2 covers the necessary thermal relations for the development of the HAM. Section 3 integrates different parts of the HAM for attitude estimation. Simulation, evaluation and verification of the HAM for an Earth-orbiting cubic satellite is discussed in Section 4. Finally, Section 5 concludes the paper, and addresses future researches currently under development.

\section{Main relations of radiation in space for the design of HAM}

One of the major characteristics of space environment is its high vacuum. Hence, for most high orbiting satellites, the residual atmospheric pressure and the resulting drag will be negligible for the problem of attitude estimation. Therefore, the aerodynamic heating and convective interaction between the satellite and the space environment can be ignored [33].

Since the radiation is the key mean of heat exchange in space between a satellite and its surrounding, it can be characterized by the following factors (see Fig. 1). 
- Radiation from the satellite to deep space.

- Direct solar radiation.

- Albedo radiation.

- Planetary (Earth) radiation.

\subsection{Radiation emission from a satellite}

Radiation is usually related to heat exchange through electromagnetic energy (with wavelength ranging from 0.1 to $100 \mu \mathrm{m}$ ) between a surface and the surrounding environment [23]. All objects with a temperature more than absolute zero emit radiation rates proportional to the fourth power of their temperature. The most efficient emitter surface is called a black surface $b$. Radiation emission, denoted by $q_{b}^{e}\left(\mathrm{~W} / \mathrm{m}^{2} \equiv\right.$ Watt per unit Area), from a black surface at temperature $T$ ( $\mathrm{K} \equiv$ Kelvin) is expressed by the Stefan-Boltzmann law:

$q_{b}^{e}=\sigma T^{4}$

or

$Q_{b}^{e}=A^{r} \sigma T^{4}$

where $\sigma=5.67 \times 10^{-8} \mathrm{~W} / \mathrm{m}^{2} \mathrm{~K}^{4}$ is the Stefan-Boltzmann constant and $A^{r}\left(\mathrm{~m}^{2}\right)$ is the radiating area.

Radiation emission from a nonblack surface $n b$ with similar area and temperature is expressed by:

$Q_{n b}^{e}=\varepsilon A^{r} \sigma T^{4}$

where $\varepsilon<1$ is known as the total hemispherical (or average) emissivity, which is the ratio of energy emission by the nonblack surface $n b$ to that of the black surface $b$ at the same temperature [23].

Considering now two black surfaces, the amount of radiation heat exchange between them depends on their temperatures and how they view one another. The visibility of one surface as seen from the other is referred to a view (shape) factor. It defines the fraction of radiation leaving one surface and intercepted by another. The net radiation from a black surface element $d A_{1}$ at temperature $T_{d A_{1}}$ to another interacting black surface element $d A_{2}$ at temperature $T_{d A_{2}}$ is given by [23]:

$d^{2} Q_{d A_{1}-d A_{2}}^{r}=\left(\sigma T_{d A_{1}}^{4}-\sigma T_{d A_{2}}^{4}\right) \cos \theta_{1}^{\prime} \cos \theta_{2}^{\prime} d A_{1} d A_{2} / \pi s^{2}$

This results in a net radiation from $A_{1}$ to $A_{2}$, which is given by:

$Q_{A_{1}-A_{2}}^{r}=\iint_{A_{1} A_{2}}\left(\sigma T_{d A_{1}}^{4}-\sigma T_{d A_{2}}^{4}\right) \frac{\cos \theta_{1}^{\prime} \cos \theta_{2}^{\prime} d A_{1} d A_{2}}{\pi s^{2}}$

where $\theta_{1}^{\prime}$ and $\theta_{2}^{\prime}$ are the angles between surface normal vectors (of elements $d A_{1}$ and $d A_{2}$ ) and the line, of length $s$, that connects them (see Fig. 2). The superscript $r$ indicates net radiation exchange, while the superscript $e$ denotes the emission alone.

Assuming isothermal conditions for the two surfaces, the net radiation $Q_{12}^{r}$ from surface 1 can be written in the following form:

$Q_{12}^{r}=F_{12} A_{1}\left(\sigma T_{1}^{4}-\sigma T_{2}^{4}\right)$

where $F_{12}$ is the shape factor expressed by [23]:

$F_{12}=\left(\frac{1}{A_{1}}\right) \iint_{A_{1} A_{2}} \frac{\cos \theta_{1}^{\prime} \cos \theta_{2}^{\prime}}{\pi s^{2}} d A_{1} d A_{2}$

If $d A_{1}$ and $d A_{2}$ are considered as the elements on the satellite and the Earth, respectively, the integration of Eq. (7) yields the required shape factors which are shown in Fig. 3.

According to Fig. 3, shape factors are determined using a parameter, $v=a / R$ in which $a$ and $R$ are the satellite height and Earth radius respectively.

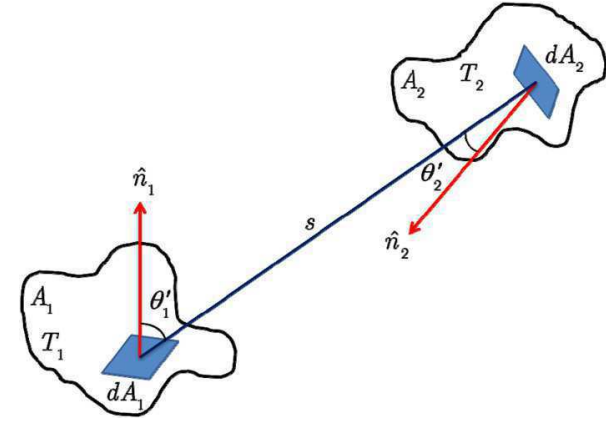

Fig. 2. Radiation heat exchange between two surfaces.

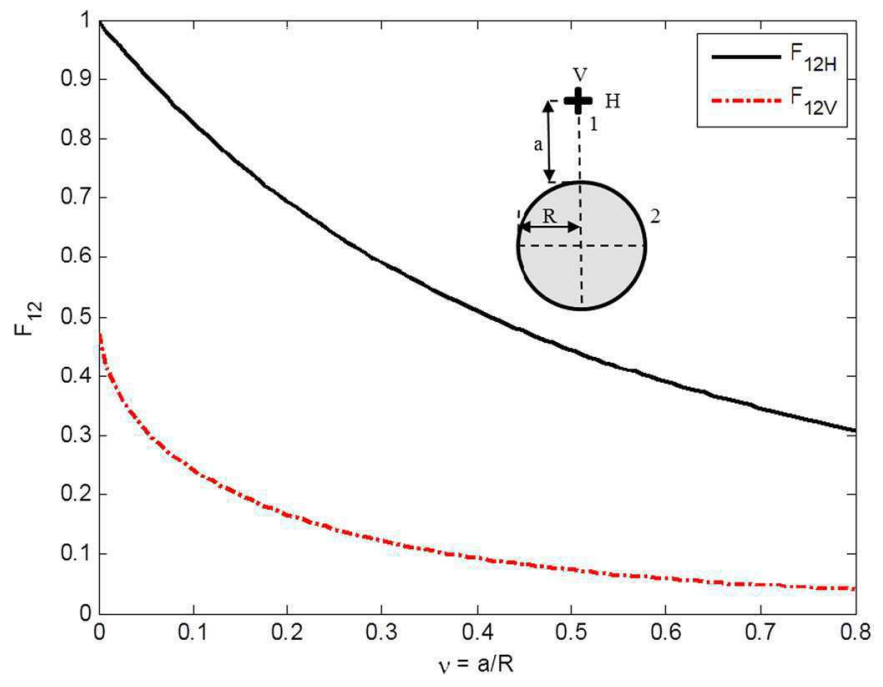

Fig. 3. Shape factors from a surface element to a sphere [23].

\subsection{Solar radiation flux}

Most measurements above the Earth's atmosphere have expressed a solar radiation flux value between 1365 and $1373 \mathrm{~W} / \mathrm{m}^{2}$ for LEO satellites. Solar radiation exists within a broad range of electromagnetic spectrum that includes about $7 \%$ in the ultraviolet (UV) wavelength $(0.31-0.40 \mu \mathrm{m}), 46 \%$ visible $(0.40-0.69 \mu \mathrm{m})$, and $47 \%$ infrared (IR) radiation (above $0.70 \mu \mathrm{m}$ ). Since solar IR has shorter wavelength against the satellite emitted IR at normal satellite temperatures, it can be utilized for conditioning of a surface to have high reflectivity in the solar spectrum with high emissivity in IR at the same time. This property is reflected in solar absorptivity, $\alpha^{S}$, which is the fraction of straight solar energy absorbed by a surface. Therefore, the absorbed solar energy $S^{\alpha}$ for a surface, whose normal vector forms an angle $\beta$ with Sun direction, can be computed using Eq. (8):

$S^{\alpha}=\alpha^{S} S \cos \beta$

where $S$ is the solar flux.

\subsection{Albedo radiation flux}

The reflected heating sunlight from the Earth surface is called albedo. Usually, it is considered as a fraction of the solar constant and can be written as:

$Q_{\text {Albedo }}^{\prime}=f_{a} S$

where $f_{a}$ is known as the albedo factor. 


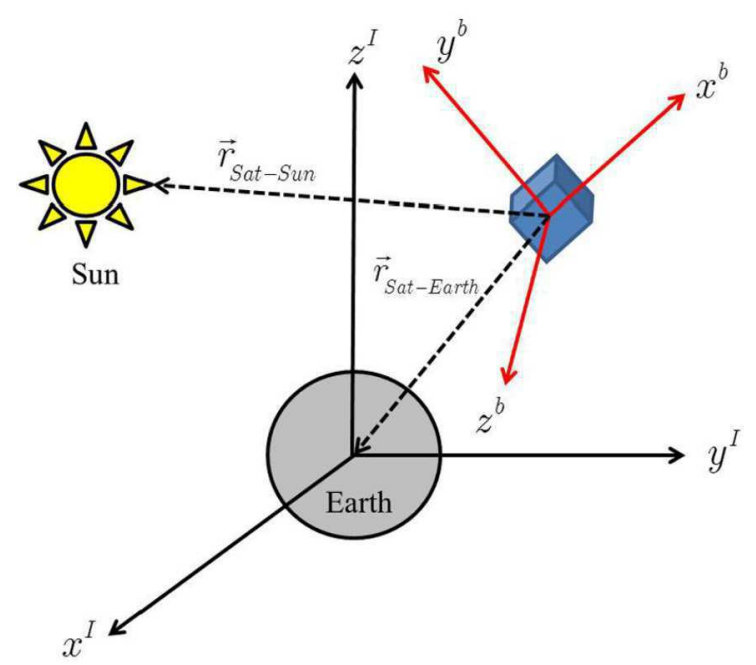

Fig. 4. Orientation of a typical satellite with respect to Sun and Earth.

Albedo appears more significant at the Earth's polar ice caps, and can be estimated in those regions with some accuracy as a function of Sun's elevation and the satellite orbital parameters. However, predictions for overland and above oceans are distorted by variable effects of cloud formations and water distribution in the atmosphere.

\subsection{Earth radiation flux}

Emitted radiation from the Earth is considered equivalent in intensity and wavelength to the heat transferred from a black surface at $-20^{\circ} \mathrm{C}$, which gives a nominal value of Earth flux approximately equal to $236 \mathrm{~W} / \mathrm{m}^{2}$. For this value, a tolerance of $\pm 38 \mathrm{~W} / \mathrm{m}^{2}$ (16\%) is usually imposed in the satellite thermal analysis [22,23]. Since the Earth radiation is in the same band as that normally emitted by satellites, the fraction of impinging Earth flux absorbed by the satellite radiator is given by its emissivity $\varepsilon$. Hence, a surface treatment intended to reflect the Earth radiation will also reduce the space radiation through the same proportion as the surface's emission ability.

\section{Design of heat-attitude model (HAM)}

In order to establish a direct relation between the received heat fluxes by the surface of a typical satellite and its corresponding attitude, the orientation of a satellite in space thermal environment is presented in Fig. 4, where $\vec{r}_{\text {Sat-Sun }}$ and $\vec{r}_{\text {Sat-Earth }}$ denote the known satellite relative position vectors with respect to Sun and Earth in the ECI respectively.

The ultimate purpose is to find the attitude of the satellite with respect to ECI. Since the normal vectors of satellite surfaces that receive the heat fluxes are defined in body coordinate system, the relation between these vectors and satellite position vectors $\left(\vec{r}_{\text {Sat-Sun }}, \vec{r}_{\text {Sat-Earth }}\right)$ can be established using shape factors computed along the parallel and the perpendicular directions with respect to the satellite position vectors. This concept is further clarified through Fig. 5.

As it is shown in Fig. 5 , the unit vector $\vec{k}$ could be either in the direction of the satellite-Earth or satellite-Sun position vectors depending on the heat source. Therefore, $\vec{k}$ can be expressed as:

$\vec{k}=\frac{\vec{r}_{\text {Sat-Earth }}}{\left\|\vec{r}_{\text {Sat-Earth }}\right\|}$ or $\quad \vec{k}=\frac{\vec{r}_{\text {Sat-Sun }}}{\left\|\vec{r}_{\text {Sat-Sun }}\right\|}$

While the distance between an Earth-orbiting satellite and the Sun is considerable, the satellite-Sun position vector is assumed

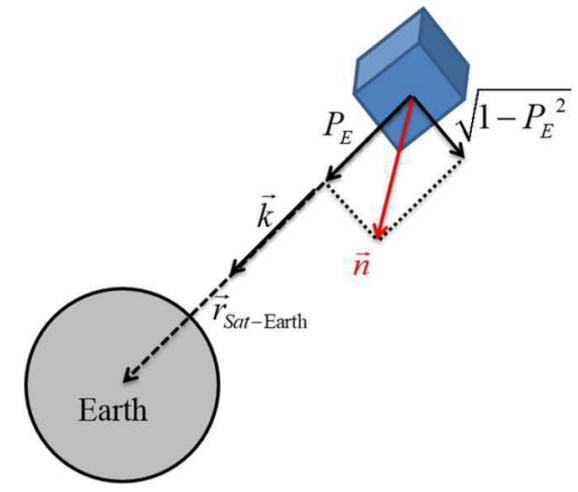

Fig. 5. Decomposition of the satellite surface normal vector $\vec{n}$.

to contain only the parallel component and thus the perpendicular component is neglected.

On the other hand, the relation between the ECI and the satellite coordinate system is also established through a rotation matrix, for which various attitude representations are possible. Considering the Euler's angles representation, the inertial to body rotation matrix is expressed as [34]:

$$
\begin{aligned}
& C_{I}^{B}= \\
& {\left[\begin{array}{ccc}
\cos \theta \cos \psi & \cos \theta \sin \psi & -\sin \theta \\
\sin \phi \sin \theta \cos \psi-\cos \phi \sin \psi & \sin \phi \sin \theta \sin \psi+\cos \phi \cos \psi & \sin \phi \cos \theta \\
\cos \phi \sin \theta \cos \psi+\sin \phi \sin \psi & \cos \phi \sin \theta \sin \psi-\sin \phi \cos \psi & \cos \phi \cos \theta
\end{array}\right]}
\end{aligned}
$$

where $\phi, \theta, \psi$ are the Euler's angles. $C_{I}^{B}$ is only a function of the Euler's angles to be determined.

\subsection{NHF decomposition}

The NHF for each surface of a satellite can be represented as follows:

$Q_{\text {net }}=Q-Q_{\text {lost }}$

where $Q$ is the total heat received from three thermal sources, i.e.

$Q=Q_{\text {sun }}+Q_{\text {Earth }}+Q_{\text {Albedo }}$

and $Q_{\text {lost }}$ is the emitted heat from satellite surfaces to the space environment that can be computed via Eq. (3).

Using the satellite-Earth and satellite-Sun position vectors, the heat can be represented as follows:

$Q_{\text {Sun }}=A \alpha^{S} S \cos \beta=A \alpha^{S} S \vec{n} \cdot \frac{\vec{r}_{\text {Sat-Sun }}}{\left\|\vec{r}_{\text {Sat-Sun }}\right\|}$
$Q_{\text {Earth }}=A G \varepsilon\left(F_{h} P_{E}+F_{v} \sqrt{1-P_{E}^{2}}\right)$

where $G$ is the Earth flux and $F_{h}$ and $F_{v}$ are the shape factors parallel and perpendicular to the satellite-Earth position vector, respectively to be determined from Fig. 3. $P_{E}$ is the projection of the satellite surface normal unit vector along the direction of the satellite-Earth position vector, and is defined as:

$P_{E}=\vec{n} \cdot \frac{\vec{r}_{\text {Sat-Earth }}}{\left\|\vec{r}_{\text {Sat-Earth }}\right\|}$

For the reflective albedo, that is a fraction of direct solar radiation in the direction of the satellite-Earth position vector, the heat can be written as [35]:

$Q_{\text {Albedo }}=A f_{a} S \alpha^{S}\left(F_{h} P_{E}+F_{v} \sqrt{1-P_{E}^{2}}\right) \cos \theta_{A}$ 


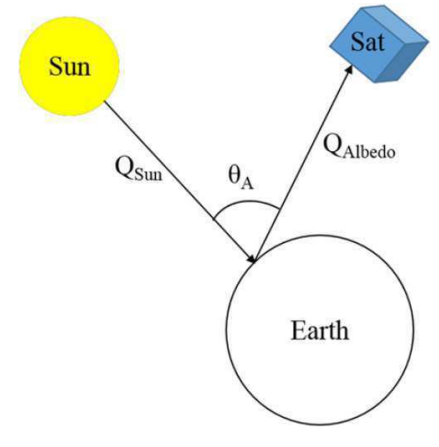

Fig. 6. Reflection of solar ray from the Earth surface [35].

in which $\theta_{A}$ is the angle between the Sun-Earth vector and albedo reflection, see Fig. 6 .

\subsection{HAM for satellite attitude estimation}

In order to establish the proposed HAM in a form of measurement equation required for estimation problem, we consider the relation between heat and temperature for each satellite surface:

$Q_{i}=m_{i} c_{i} \frac{d T_{i}}{d t}$

where $i=1,2,3$ denotes the number of orthogonal satellite surfaces, $m_{i}$ and $c_{i}$ are the mass and specific heat capacity of each surface respectively.

Based on Eq. (12) and Eq. (18), the temperature rate for each satellite surface will be:

$\frac{d T_{i}}{d t}=\frac{Q_{n e t}}{m_{i} c_{i}}$

On the other hand, substituting Eq. (12) through Eq. (17) into Eq. (19), yields the temperature rate of each orthogonal satellite surfaces:

$$
\begin{aligned}
\frac{d T_{i}}{d t}= & \frac{1}{m_{i} c_{i}}\left[A_{i} \alpha_{i}^{S} S \vec{n}_{i} \cdot C_{I}^{B} \frac{\vec{r}_{\text {Sat-Sun }}^{I}}{\left\|\vec{r}_{\text {Sat-Sun }}^{I}\right\|}\right. \\
& +A_{i} G \varepsilon_{i}\left(F_{h} P_{E_{i}}+F_{v} \sqrt{1-P_{E_{i}}^{2}}\right) \\
& \left.+A_{i} f_{a} S \alpha_{i}^{S}\left(F_{h} P_{E_{i}}+F_{v} \sqrt{1-P_{E_{i}}^{2}}\right) \cos \theta_{A}-A_{i} \varepsilon_{i} \sigma T_{i}^{4}\right]
\end{aligned}
$$

Finally, using the inertial to body transformation matrix, $C_{I}^{B}$ from Eq. (11) into Eq. (20) complete the HAM for three satellite orthogonal surfaces,

$$
\begin{aligned}
\frac{d T_{1}}{d t}= & \frac{A_{1}}{m_{1} c_{1}}\left\{-\varepsilon_{1} \sigma T_{1}^{4}+S \alpha_{1}^{S}\left[r_{S_{x}} \cos (\phi) \cos (\theta)-r_{S_{z}} \sin (\theta)\right.\right. \\
& \left.\left.+r_{S_{y}} \cos (\theta) \sin (\psi)\right]+a_{1}\left[G \varepsilon_{1}+S f_{a} \alpha_{1}^{S} \cos \left(\theta_{A}\right)\right]\right\} \\
a_{1}= & F_{v} \sqrt{1-\left[r_{E_{x}} \cos (\psi) \cos (\theta)-r_{E_{z}} \sin (\theta)+r_{E_{y}} \cos (\theta) \sin (\psi)\right]^{2}} \\
+ & F_{h}\left[r_{E_{x}} \cos (\psi) \cos (\theta)-r_{E_{z}} \sin (\theta)+r_{E_{y}} \cos (\theta) \sin (\psi)\right]
\end{aligned}
$$

$$
\begin{aligned}
\frac{d T_{2}}{d t}= & \frac{-A_{2}}{m_{2} c_{2}}\left\{\varepsilon_{2} \sigma T_{2}^{4}+b_{1}\left[G \varepsilon_{2}+S f_{a} \alpha_{2}^{S} \cos \left(\theta_{A}\right)\right]\right. \\
& \left.+S \alpha_{2}^{S}\left[-r_{S_{x}} b_{3}+r_{S_{y}} b_{4}+r_{S_{z}} \cos (\theta) \sin (\phi)\right]\right\}
\end{aligned}
$$

$$
\begin{aligned}
b_{1} & =F_{h} b_{2}+F_{v} \sqrt{1-b_{2}^{2}} \\
b_{2} & =-r_{E_{x}} b_{3}+r_{E_{y}} b_{4}+r_{E_{z}} \cos (\theta) \sin (\phi) \\
b_{3} & =\cos (\phi) \sin (\psi)-\cos (\psi) \sin (\phi) \sin (\theta) \\
b_{4}= & \cos (\phi) \cos (\psi)+\sin (\phi) \sin (\psi) \sin (\theta) \\
\frac{d T_{3}}{d t}= & \frac{A_{3}}{m_{3} c_{3}}\left\{\varepsilon_{3} \sigma T_{3}^{4}+c_{1}^{\prime}\left[G \varepsilon_{3}+S f_{a} \alpha_{3}^{S} \cos \left(\theta_{A}\right)\right]\right. \\
& \left.\quad+S \alpha_{3}^{S}\left[r_{S_{x}} c_{4}^{\prime}-r_{S_{y}} c_{3}^{\prime}+r_{S_{z}} \cos (\phi) \cos (\theta)\right]\right\} \\
c_{1}^{\prime}= & F_{h} c_{2}+F_{v} \sqrt{1-c_{2}^{\prime 2}} \\
c_{2}^{\prime}= & r_{E_{x}} c_{4}-r_{E_{y}} c_{3}+r_{E_{z}} \cos (\phi) \cos (\theta) \\
c_{3}^{\prime}= & \cos (\psi) \sin (\phi)-\cos (\phi) \sin (\psi) \sin (\theta) \\
c_{4}^{\prime}= & \sin (\phi) \sin (\psi)+\cos (\phi) \cos (\psi) \sin (\theta)
\end{aligned}
$$

where, $r_{S_{x}}, r_{S_{y}}, r_{S_{z}}$ are the components of $\vec{r}_{\text {Sat-Sun }}^{I}$ vector and, similarly, $r_{E_{x}}, r_{E_{y}}, r_{E_{z}}$ are the components of $\vec{r}_{\text {Sat-Earth }}^{I}$ vector, described previously.

In general, the measurement equation takes usually the following form:

$z=h(x)+v$

where $h$ (Eq. (21) through Eq. (23)) relates the measurements $z$ (temperature rates) to the states $x$ (attitude) and $v$ is the sensor measurement noise, which is assumed to be a white Gaussian noise.

Therefore, by considering Eq. (21) through Eq. (23) and Eq. (24), the measurement vector is composed of the temperature rates (to be measured via temperature sensors in appropriate time intervals):

$z=\left[\begin{array}{lll}\frac{d T_{1}}{d t} & \frac{d T_{2}}{d t} & \frac{d T_{3}}{d t}\end{array}\right]^{T}+v$

\subsection{Results and discussion}

To resume, the following summary establishes clearly the relation between the NHF and the satellite's Attitude.

- Based on Eqs. (14), (15) and (17), the received heat is related to the shape factors and the components of $\vec{n}$ for each surface of the satellite. Note that the satellite relative position vectors toward the major heat sources (Sun and Earth) are known in ECI.

- The body components of each surface normal unit vector is considered as a row in the transformation matrix, namely $C_{I}^{B}$ to be determined (see Eq. (11)).

- Since the nine elements of the rotation matrix can be written in terms of three independent variables (namely the Euler's angles) that are explicitly present in Eq. (21) through Eq. (23), there are three equations and three unknowns to be determined.

- Solution of this system of equations will deterministically establish the satellite attitude using the proposed HAM.

It is important to emphasize that the key contribution of this paper is to develop a novel HAM and verify its reliability for an Earth-orbiting satellite. The verification process, which requires the temporal variation of the rotation matrix for continuous attitude determination, is accomplished using the satellite attitude dynamics and will be discussed in the next section. 
Table 1

Satellite orbital parameters.

\begin{tabular}{ll}
\hline Eccentricity (e) & 0 \\
Right ascension $(\Omega)$ & $60^{\circ}$ \\
Inclination (i) & $40.5^{\circ}$ \\
Argument of perigee $(\omega)$ & $270^{\circ}$ \\
Orbital height & $460 \mathrm{~km}$ \\
\hline
\end{tabular}

Table 2

Satellite system features.

\begin{tabular}{ll} 
Satellite mass & $49.86 \mathrm{~kg}$ \\
Satellite volume & $1 \mathrm{~m} \times 1 \mathrm{~m} \times 1 \mathrm{~m}$ \\
Initial angular velocity $\left(\omega_{0}\right)$ & {$\left[\begin{array}{lll}0 & -0.063 & 0\end{array}\right]^{T} \mathrm{deg} / \mathrm{sec}$} \\
Initial Euler angles & {$\left[\begin{array}{lll}-49.5 & 0 & 60\end{array}\right]^{T} \mathrm{deg}$} \\
\hline
\end{tabular}

Table 3

Satellite thermal model properties.

\begin{tabular}{ll}
\hline Solar radiation heat flux & $1414 \mathrm{~W} / \mathrm{m}^{2}$ \\
Earth radiation heat flux & $257 \mathrm{~W} / \mathrm{m}^{2}$ \\
Solar absorptivity & 0.33 \\
Albedo factor & 0.26 \\
Surface material & Aluminium \\
Heat specific capacity & $961 \mathrm{~J} / \mathrm{kg} \mathrm{K}$ \\
Surface emissivity factor & 0.509 \\
\hline
\end{tabular}

\section{Verification of heat-attitude model}

For the purpose of this paper, the Euler's angles representation is chosen to express the rotational kinematics of the satellite. Through the use of the Euler's equations (26) and the kinematic equations (27), the satellite rotational dynamics and attitude can be written as [36]:

$\dot{\omega}_{x}=\frac{M_{x}-\omega_{y} \omega_{z}\left(I_{z}-I_{y}\right)}{I_{x}}$

$\dot{\omega}_{y}=\frac{M_{y}-\omega_{x} \omega_{z}\left(I_{x}-I_{z}\right)}{I_{y}}$

$\dot{\omega}_{z}=\frac{M_{z}-\omega_{x} \omega_{y}\left(I_{y}-I_{x}\right)}{I_{z}}$

$\dot{\phi}=\omega_{x}+\left[\omega_{y} \sin (\phi)+\omega_{z} \cos (\phi)\right] \tan (\theta)$

$\dot{\theta}=\omega_{y} \cos (\phi)-\omega_{z} \sin (\phi)$

$\dot{\psi}=\left[\omega_{y} \sin (\phi)+\omega_{z} \cos (\phi)\right] \sec (\theta)$

where $\omega=\left[\begin{array}{lll}\omega_{x} & \omega_{y} & \omega_{z}\end{array}\right]^{T}$ is the angular velocity of the satellite expressed in the body coordinate system.

By considering Eq. (26) and Eq. (27) and using Eq. (21) through Eq. (23), the temperature, as well as the NHF that each surface of satellite receives, will be attainable. Table 1 presents the orbital characteristics used for the simulation of HAM. In addition, Table 2 provides some system features required for the simulations that are performed using MATLAB [37], as well as the initial states of the nadir pointing satellite. Specific surface features and preparations required for proper applicability of the developed HAM are beyond the scope of this work. However, some of the thermal data which are used in thermal part of the model are presented in Table 3. The results of simulations using the data of Tables 1,2 and 3 are presented in Figs. 7-10.

In order to partially verify the proposed HAM, commercial thermal analysis tools are used for comparison of results. Thermal Desktop [38] and SINDA [39] are used to obtain the NHFs and the satellite surface temperatures, respectively. For comparative purposes, the temperature of three orthogonal surfaces resulted out of HAM are compared with those of the Thermal Desktop in Fig. 11 through Fig. 13, whose trends and accuracy are well compatible.
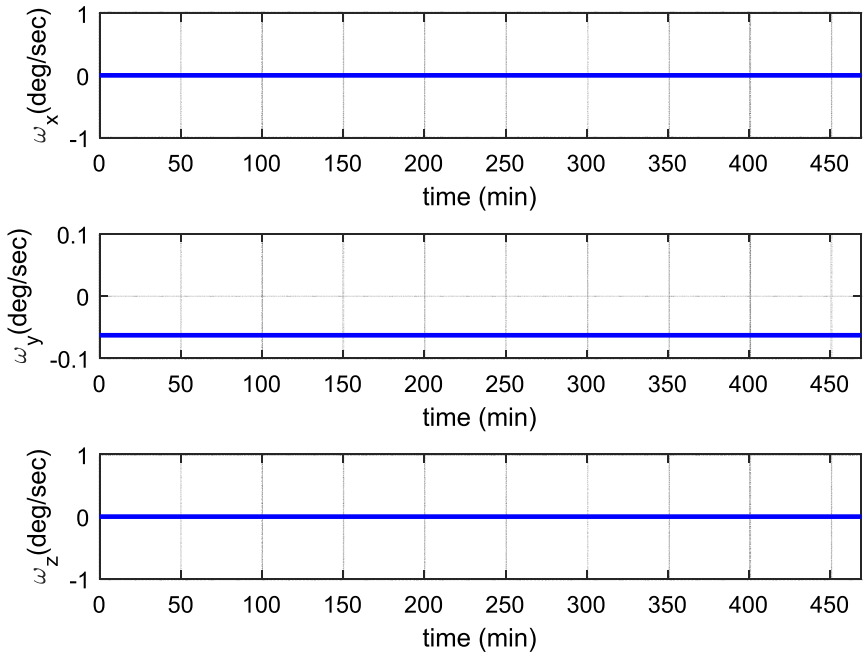

Fig. 7. Satellite angular velocities.
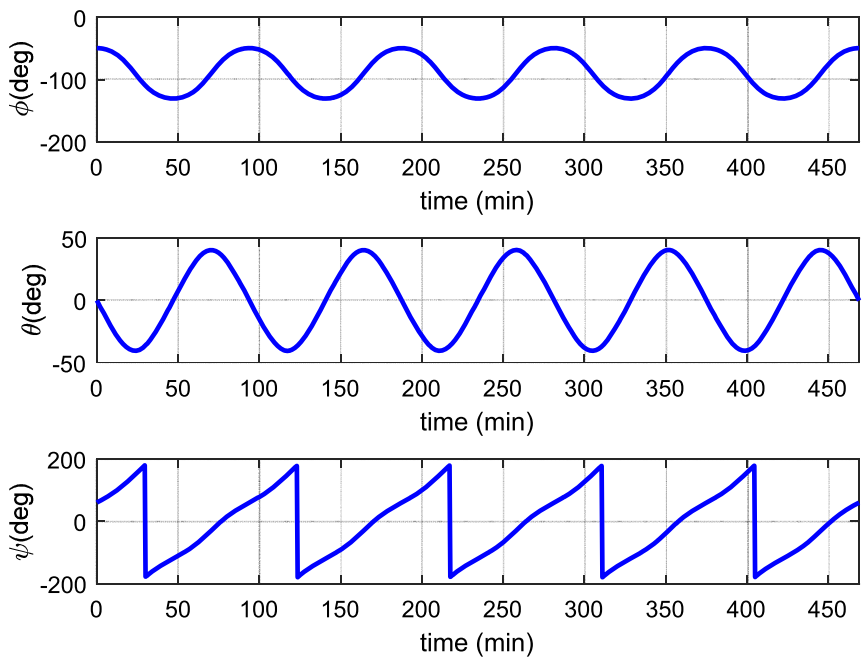

Fig. 8. Satellite Euler angles.
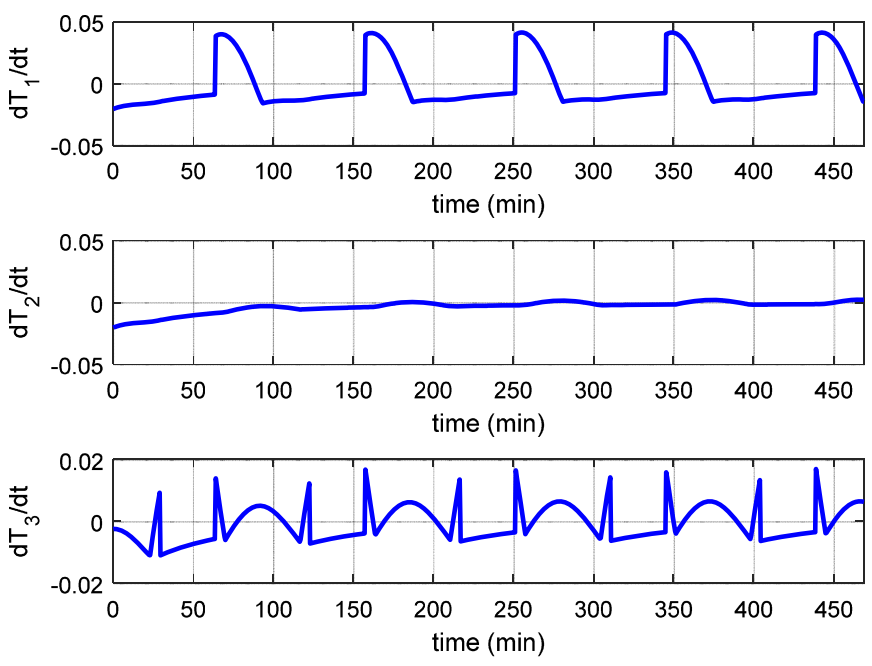

Fig. 9. Temperature rates for three orthogonal surfaces. 

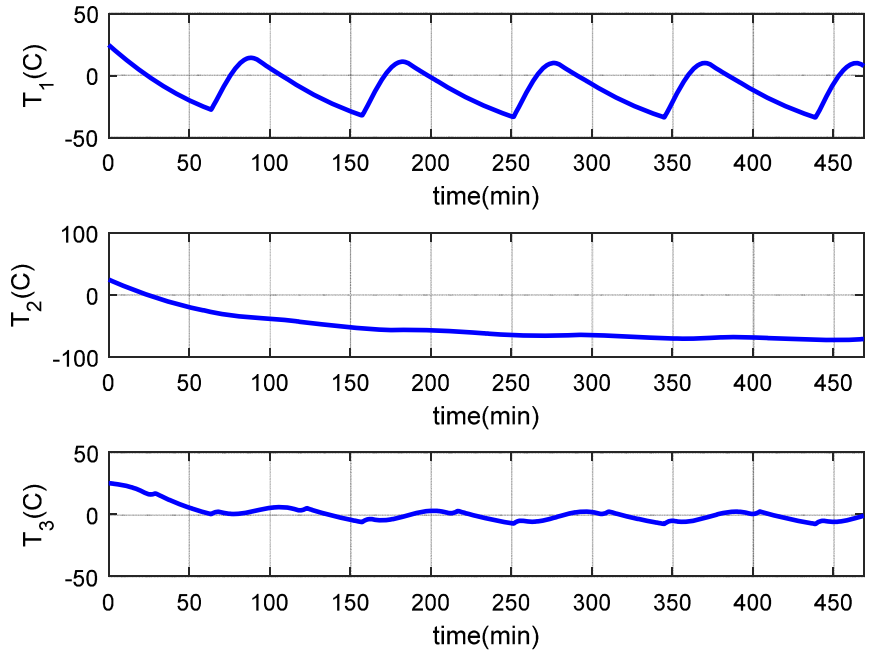

Fig. 10. Satellite surface temperatures.

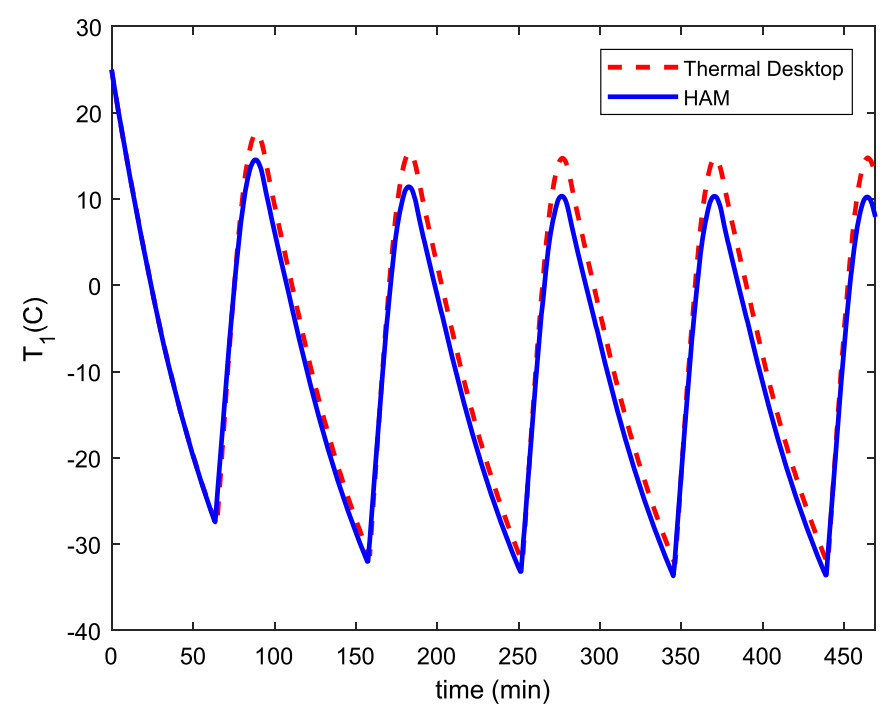

Fig. 11. Temperature variations of surface 1.

As shown in Figs. 11 through 13, the HAM surface temperature results closely follow those obtained via the thermal analysis tools with good accuracy. This observation verifies the HAM appropriateness to represent a measurement model for AD stochastic formulation. Based on the above results and those presented in [30] and [31], a performance comparison is presented between the proposed HAM package against the other existing AD systems in Table 4.

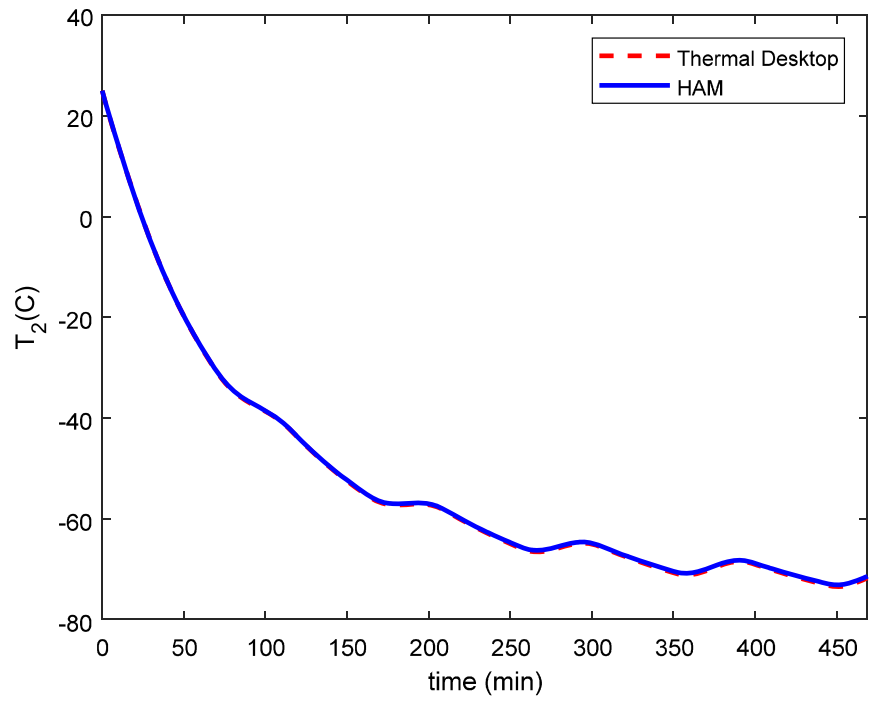

Fig. 12. Temperature variations of surface 2 .

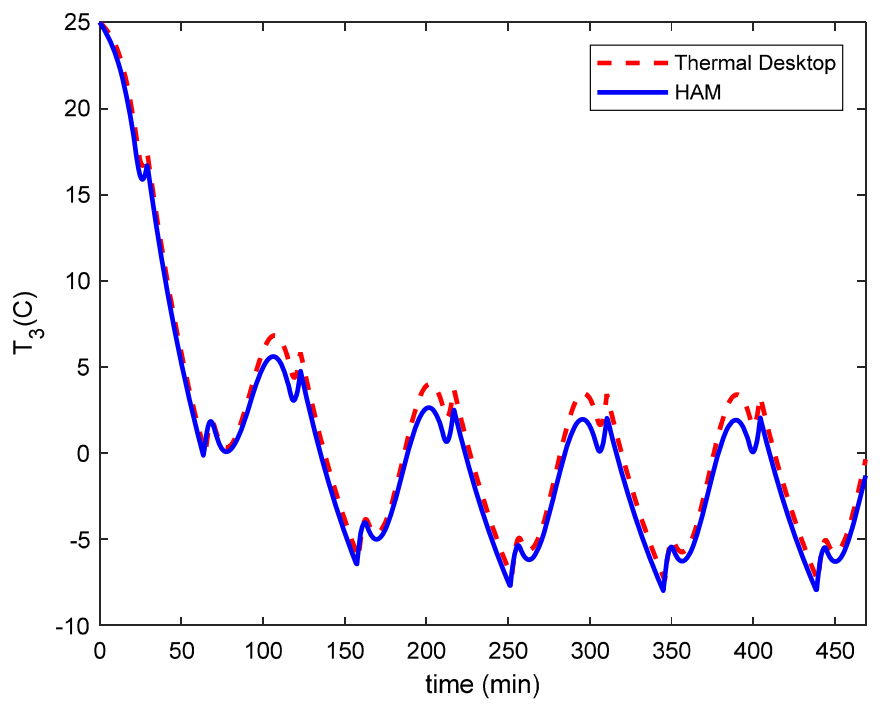

Fig. 13. Temperature variations of surface 3 .

\section{Conclusion}

A novel radiation based heat model for attitude estimation of satellites is proposed in this paper. The heat-attitude model (HAM) uses external radiating heat sources affecting the satellite external surface temperatures to produce the net heat flux (NHF) that each satellite surface receives. Decomposition of the NHFs in parallel and perpendicular components with respect to the heat sources allows for generation of the inertial to satellite rotation matrix

Table 4

Existing AD systems.

\begin{tabular}{|c|c|c|c|}
\hline Sensors & Typical performance range & Mass (kg) & Power (W) \\
\hline Gyroscopes & $\begin{array}{l}\text { Drift rate }=0.003 \mathrm{deg} / \mathrm{h} \text { to } 1 \mathrm{deg} / \mathrm{h} \\
\text { Drift rate stability varies widely }\end{array}$ & $<0.1$ to 15 & $<1$ to 200 \\
\hline Sun sensors & Accuracy $=0.005 \mathrm{deg}$ to $3 \mathrm{deg}$ & 0.1 to 2 & 0 to 3 \\
\hline $\begin{array}{l}\text { Star sensors } \\
\text { (Scanners and cameras) }\end{array}$ & $\begin{array}{l}\text { Accuracy }=1 \text { arcsecond to } 1 \text { arcminute } \\
(=0.0003 \text { deg to } 0.01 \mathrm{deg})\end{array}$ & 2 to 5 & 5 to 20 \\
\hline Horizon sensors & Accuracy: & 1 to 4 & 5 to 10 \\
\hline Scanner/pipper & $0.05 \mathrm{deg}$ to $1 \mathrm{deg}(0.1 \mathrm{deg}$ is best for LEO) & 0.5 to 3.5 & 0.3 to 5 \\
\hline Fixed head (static) & $<0.1 \mathrm{deg}$ to $0.25 \mathrm{deg}$ & & \\
\hline Magnetometer & Accuracy $=0.5 \mathrm{deg}$ to $3 \mathrm{deg}$ & 0.3 to 1.2 & $<1$ \\
\hline HAM package & Accuracy $=0.2 \mathrm{deg}$ to $5 \mathrm{deg}$ & $<0.05$ & $<0.05$ \\
\hline
\end{tabular}


that, in turn, leads to its attitude knowledge. Simulation results of the proposed HAM are verified via commercially available thermal analysis tools for a typical LEO satellite. The proposed HAM satellite temperature results are in good agreement with those of the available commercial thermal softwares. Thus, the proposed HAM can be considered as a viable measurement model for stochastic $\mathrm{AD}$ via a large class of filtering techniques, some of which have been already utilized by researchers.

\section{Conflict of interest statement}

The authors declare that there is no conflict of interest over this article.

\section{Acknowledgement}

Authors thank Dr. Mohsen Abedi for his consultation on thermal modelling utilized in parts of this research.

\section{References}

[1] G. Wahba, A least-square estimation of satellite attitude, Problem 65-1, SIAM Rev. 7 (3) (1965) 409.

[2] M.L. Psiaki, Global magnetometer-based spacecraft attitude and rate estimation, J. Guid. Control Dyn. 27 (2) (2004) 240-250.

[3] B. Chen, G. Yunhai, Y. Xu, High precision attitude estimation algorithm using three star trackers, in: Proceeding of the 10th World Congress on Intelligent Control and Automation, 6-8 July 2012, pp. 4168-4173.

[4] M.L. Psiaki, Autonomous low-earth-orbit determination from magnetometer and sun sensor data, J. Guid. Control Dyn. 22 (2) (1999) 296-304.

[5] K. Son-Goo, J.L. Crassidis, Y. Cheng, A.M. Fosbury, J.L. Junkins, Kalman filtering for relative spacecraft attitude and position estimation, J. Guid. Control Dyn. 30 (1) (2007) 133-143.

[6] J.C. Springmann, A.J. Sloboda, A.T. Klesh, M.W. Bennett, J.W. Cutler, The attitude determination system of the RAX satellite, Acta Astronaut. 75 (2012) 120-135.

[7] J.L. Farrell, Attitude determination by Kalman filter, Automatica 6 (5) (1970) 419-430.

[8] J.W. Murrel, Precision attitude determination for multimission spacecraft, in: AIAA Guidance and Control Conference, Palo Alto, CA, 1978.

[9] E.J. Lefferts, F.L. Markley, M.D. Shuster, Kalman filtering for spacecraft attitude estimation, J. Guid. Control Dyn. 5 (5) (1982) 417-429.

[10] F.L. Markley, Attitude error representation for Kalman filtering, J. Guid. Control Dyn. 63 (2) (2003) 311-317.

[11] M.D. Shuster, Constraint in attitude estimation, part I: constrained estimation, J. Astronaut. Sci. 51 (1) (2003) 51-74

[12] M.D. Shuster, Constraint in attitude estimation, part II: unscented estimation, J. Astronaut. Sci. 51 (1) (2003) 75-100.

[13] S.J. Julier, J.K. Uhlmann, H.F. Durrant-Whyte, A new approach for filtering nonlinear systems, in: Proccedings of the American Control Conference, Seattle, WA, 1995.

[14] E. Wan, R. Van der Merwe, The unscented Kalman filter, in: Kalman Filtering and Neural Networks, John Wiley \& Sons, New York, NY, 2001.
[15] M. Norgaard, N.K. Poulsen, O. Ravan, New development in state estimation for nonlinear systems, Automatica 36 (11) (2000) 1627-1638.

[16] J.L. Crassidis, F.L. Markley, Unscented filtering for spacecraft attitude estimation, J. Guid. Control Dyn. 26 (4) (2003) 536-542.

[17] M. Kiani, S.H. Pourtakdoust, Concurrent orbit and attitude estimation using minimum sigma point unscented Kalman filter, J. Aerosp. Eng. 228 (6) (2013) 801-819.

[18] A. Doucet, N. Freitas, N. Gordan, Sequential Monte Carlo Methods in Practice, Springer, New York, NY, 2001.

[19] M.S. Arulampalam, S. Maskell, N. Gordon, T. Clapp, A tutorial on particle filters for online nonlinear/non-Gaussian Bayesian tracking, IEEE Trans. Signal Process. 50 (2) (2002) 174-185.

[20] Y. Cheng, J.L. Crassidis, Particle filtering for sequential spacecraft attitude estimation, in: AIAA Guidance, Navigation, and Control Conference, Providence, RI, 2004.

[21] Y. Oshman, A. Carmi, Estimating attitude from vector observations using a genetic algorithm-embedded quaternion particle filter, in: AIAA Guidance, Navigation, and Control Conference, Providence, RI, 2004.

[22] D.G. Gilmor, Spacecraft Thermal Control Handbook, The Aerospace Corporation, 2002.

[23] R.D. Karam, Satellite Thermal Control for System Engineers, AIAA, 1998.

[24] J.R. Tsai, Overview of satellite thermal analytic model, J. Spacecr. Rockets 41 (1) (2004) 120-125.

[25] J. Gaite, A. Sanz-Andres, I. Perez-Grande, Nonlinear analysis of a simple mode of temperature evolution in a satellite, Nonlinear Dyn. 58 (2009) 405-415.

[26] J. Gaite, Nonlinear analysis of spacecraft thermal models, Nonlinear Dyn. 65 (2011) 283-300.

[27] N.D. Anh, N.N. Hieu, P.N. Chung, N.T. Anh, Thermal radiation analysis for small satellites with single-node model using techniques of equivalent linearization, Appl. Therm. Eng. 94 (2016) 607-614.

[28] H.B. Khaniki, S.M.H. Karimian, Determining the heat flux absorbed by satellite surfaces with temperature data, J. Mech. Sci. Technol. 28 (6) (2014) 2393-2398.

[29] H.B. Khaniki, S.M.H. Karimian, Satellite attitude determination using absorbed heat fluxes, J. Aerosp. Eng. 29 (6) (2016) 04016053.

[30] A. Labibian, A. Alikhani, S.H. Pourtakdoust, Performance of a novel heat based model for spacecraft attitude estimation, Aerosp. Sci. Technol. 70 (2017) 317-327.

[31] A. Labibian, S.H. Pourtakdoust, M. Kiani, A.A. Sheikhy, A. Alikhani, Experimenta validation of a novel radiation based model for spacecraft attitude estimation, Sens. Actuators A 250 (2016) 114-122.

[32] "products/satellite-equipment/cess-a-cess," SpaceTech GmbH, [Online]. Available: http://www.spacetech-i.com/products/satellite-equipment/cess-a-cess.

[33] P. Fortescue, G. Swinered, J. Stark, Spacecraft Systems Engineering, John Wiley \& Sons, 2011.

[34] M.J. Sidi, Spacecraft Dynamics and Control, Cambridge University Press, New York, 1997.

[35] M. Moghanipour, Modeling and Identification of Satellite Thermal Radiation Model for Attitude Estimation Using Temperature Sensors, MSc Thesis, Sharif University of Technology, Tehran, 2018.

[36] B. Wie, Spacecraft Vehicle Dynamics and Control, AIAA, 1998.

[37] MATLAB, Ver. 8.4, MathWorks, 2014.

[38] Thermal Desktop, Ver. 4.8, C\&R Technologies, 2005

[39] SINDA, Ver. 4.8, C\&R Technologies, 2005. 